\title{
Anti-inflammatory effect of Haloperidol in Rat: inhibit Activation of TLR/MD-2
}

\author{
Nima Bakhtiari ${ }^{1}$, Amir Abbasnezhad ${ }^{2}$, Siamak Asri Rezaei ${ }^{3}$, Razie Choghakhori ${ }^{2}$, Amin Hasanvand ${ }^{2}$ \\ ${ }^{I}$ Basic Pharmacology, Faculty of Pharmacy, Ahvaz Jundishapur University of medical science, Iran, ${ }^{2}$ Nutritional Health \\ Research Center, Department of Nutrition, Lorestan University of Medical Sciences, Khorramabad, Iran, ${ }^{3}$ Department \\ of Internal medicine and Clinical pathology, Faculty of Veterinary Medicine, Urmia University, Urmia, Iran
}

Introduction: Haloperidol is anti-psychotic agent that may play anti-inflammatory role. In this, study the antiinflammatory inflammation effect of haloperidol in induction by LPS (Lipopolysaccharide of E.Coli wall) was evaluated.

Material and Methods: in first experiment haloperidol was dock with LTR4/MD-2 in silico. In second study In vivo, 120 male wistar rat divide into 8 groups. Each group contain 15 male rat. 1) Hal (haloperidol) $0.5 \mathrm{mg} / \mathrm{kg}$, 2) $\mathrm{Hal} 1 \mathrm{mg} / \mathrm{kg}, 3$ ) Hal $2 \mathrm{mg}$, 4) Hal $0.5 \mathrm{mg} / \mathrm{kg}$ po- LPS ip , 5) Hal $1 \mathrm{mg} / \mathrm{kg}$ po-LPS ip, 6) Hal $2 \mathrm{mg} / \mathrm{kg}$ po-LPS ip, 7) normal saline po and ip, 8)normal saline po and LPS ip. All gavages was done one time for 7 days. Blood sample was collected 0.5, 3 and 6 hour later as the sub group. CRP, Fibrinogen and a 2Macrogloboline as index in inflammation were assessed by ELISA kit. Result: in first study in silico of docking LTR/MD-2 showed potential of inhibition LTR/MD-2/LPS complex. in vivo study haloperidol showed inhibition in of CRP elevation. This inhibition are in acute phase of inflammation was seen. Although alpha 2 Macroglubolin and fibrinogen were increased in late phase of inflammation and haloperidol inhibited increase of both, dose-dependently effect was seen in amount of a-2 Macroglubolin conclusion: haloperidol can inhibit signal transduction of LTR4/MD-2 in signaling of inflammation. 